Z Epileptol 2013 · 26:197-197

DOI 10.1007/s10309-013-0362-y

Online publiziert: 18. Oktober 2013

(c) Springer-Verlag Berlin Heidelberg 2013

B.J. Steinhoff ${ }^{1}$ H. Potschka ${ }^{2}$

${ }^{1}$ Epilepsiezentrum Kork, Kehl-Kork

2 Institut für Pharmakologie, Toxikologie und Pharmazie, Ludwig-Maximilians-Universität, München

\title{
Breites Spektrum innerhalb der Epileptologie
}

Liebe Leser der Zeitschrift für

Epileptologie,

es ist für uns sehr erfreulich, dass wir als letztes Heft des Jahrgangs 2013 es uns als Herausgeber leisten können, ein freies Heft zu konzipieren, das nahezu ausschließlich aus frei eingereichten Beiträgen besteht. Ein Übersichtsartikel von C. Baumgartner aus Wien gehört inhaltlich noch zu dem State-of-the-Art-Heft 03/2013. Die Arbeiten decken naturgemäß ein breites Spektrum innerhalb der Epileptologie ab und werden hoffentlich auf Ihr Interesse stoßen. Wir sind hochgradig daran interessiert, auch weiterhin frei eingereichte Beiträge für die Zeitschrift für Epileptologie zu erhalten. Die nächsten Hefte sind wiederum als Schwerpunkthefte konzipiert. Unter anderem werden Stimulationstechniken, pädiatrische Themen und Stoffwechselerkrankungen adressiert werden.

Wir danken allen Lesern, Abonnenten und Autoren ebenso wie der Redaktion und dem Verlag für die großartige Unterstützung.

Auch danken wir sehr herzlich gerade angesichts des deutlich reduzierten Engagements den noch verbliebenen Sponsoren entsprechender Inserate. Man lernt dadurch, welche Industriepartner wirkliche Partner der Epileptologie sind. Trotz allem freuen wir uns auf das kommende Jahr und eine hoffentlich dann auch wieder wissenschaftlich sowie informativ ansprechende Zeitschrift für Epileptologie.

H. Potschka, München

B.J. Steinhoff, Kehl-Kork

\section{Korrespondenzadresse}

Prof. Dr. B.J. Steinhoff

Epilepsiezentrum Kork

Landstr. 1, 77694 Kehl-Kork

bsteinhoff@epilepsiezentrum.de

\section{Einhaltung ethischer Richtlinien}

Interessenkonflikt. B.J. Steinhoff und H. Potschka geben an, dass kein Interessenkonflikt besteht.

Das vorliegende Manuskript enthält keine Studien an Menschen oder Tieren. 